chain, Gly-Trp changes are bound to be rare.

There is a strong preference for mutation of the first rather than the second base of the glycine codon (41/53) and a marginal preference for mutations in $\mathrm{CpG}$ dinucleotides which are frequent targets in other genes. For a $\mathrm{CpG}$ pair to involve a glycine codon, the final base of the preceding codon must be a $\mathrm{C}$ and, although this is unusual (47/591 ( 8 per cent) in genomic DNA), 8 of the 41 first-base substitutions (19.5 per cent) occur in CpG pairs.

The enormous variation in the OI phenotype is one of its most intriguing features. It varies from forms that are lethal in the perinatal period, through crippling but non-lethal, to a comparatively mild disease where there is only a marginally increased tendency for bones to fracture. Examples from the entire range are found among the axial glycine mutants, so the question arises: how can the dramatically different effects of what are, on the face of it, fairly similar mutations be best explained? The three variables to play with are chain $(\alpha 1$ or $\alpha 2)$, the substituted residue and its position.

Taking position first, even three years ago (see my previous News and Views ${ }^{1}$ "Genetics cracks bone disease") it looked as though glycine substitutions had a more severe effect on the phenotype when they occurred towards the C-terminal end of the helix. By and large, that pattern has been adhered to by subsequent mutations found in the $\alpha 1$ chain, though there are exceptions. The correlation in $\alpha 2$ is much weaker. For instance a Gly-Cys mutation at position 646 was found in a fairly mild case, whereas an upstream Gly-Cys mutation at position 259 produced a much more severe phenotype (R. Wenstrup, Duke University) and at position 472 it was lethal. Generally, the $\alpha 1$ mutants are more damaging than their equivalents in the $\alpha 2$ chain. Nobody at the meeting disagreed with the reasoning that because there were two $\alpha 1$ chains and one $\alpha 2$ in each molecule, the simple stoichiometry of their construction would leave only one-quarter of molecules without a mutant chain if it were an $\alpha 1$ but one-half of molecules free of a mutant subunit if it were an $\alpha 2$.

The substituted residue also has some correlation with phenotype, so bulky aspartate is lethal wherever it occurs in the axial position, whereas arginine and cysteine are comparatively mild towards the $\mathrm{N}$ terminus. The pattern was all beginning to look a bit cosy until two Gly-Ser mutations at positions 832 and 844 in $\alpha 1$, which should have been lethal, were described from severely disabled but nonetheless surviving patients ( $\mathrm{P}$. Byers, University of Washington, Seattle). Rationalists blamed the presence of local 'permissive' regions for helix unfolding, but there doesn't seem to be anything remarkable about the sequences surrounding positions 832 or 844 .

That some patients with the more $\mathrm{N}$ terminal mutations lacked fractures, but still had overall osteoporosis and scoliosis, tempted some to blur the boundary between rare and common disease by claiming an OI-style mutation ( $\alpha 2$ GlyArg 661) for a patient not with OI but with post-menopausal osteoporosis (D. Prockop). If similar mutations really are a frequent cause of such a common disease then the implications are obviously enormous. Until we know, there is a danger that such claims and others like it (for example Arg-Cys 591 in the related type-II cartilage collagen in osteoarthritis-chondrodysplasia ${ }^{2}$ ) might be mere semantic exercises.

Axial glycines apart, the other feature of collagen that makes it a sitting duck for mutation is the hundred or so intron-exon boundaries that have to be kept in good shape. The trickle of deletion mutants being discovered three years ago has become, if not a flood, then a steady stream. Multi-exon deletions seem to occur rarely, always result in a lethal phenotype and are true genomic deletions. More commonly, single exons are missing from the messenger RNA, not as a result of genomic deletions but because of mutations in the splice-donor or acceptor sites. Those readers reared on globin might expect such errors to result in a failure to excise the mutant intron or the activation of cryptic sites. Not so for collagen. What happens is neat excision of the exon and its flanking introns, perhaps by a mechanism (D. Rowe, University of Connecticut) based on a recent model for exon definition ${ }^{3}$. After interaction at the $3^{\prime}$ end, correct assembly of the spliceosome depends on a donor site being within 300 base pairs (bp) downstream. If the wild-type site on the other side of the exon is mutated and no other site is found within $300 \mathrm{bp}$ then that interval is not recognized as an exon. Processing then excises the whole stretch between the intact upstream donor and downstream acceptor sites, including the now invisible exon.

Nine single-exon skips due to boundary mutations were described in $\alpha 1$ and seven in $\alpha 2$. Because the triplet frame is left intact, molecules can form and the $\alpha 1$ and $\alpha 2$ stoichiometry effect comes into play. All the $\alpha 1$ skip mutations are effectively lethal (a patient carrying one is still alive thanks only to intensive care), with the

\title{
Good vibrations from a violin
}
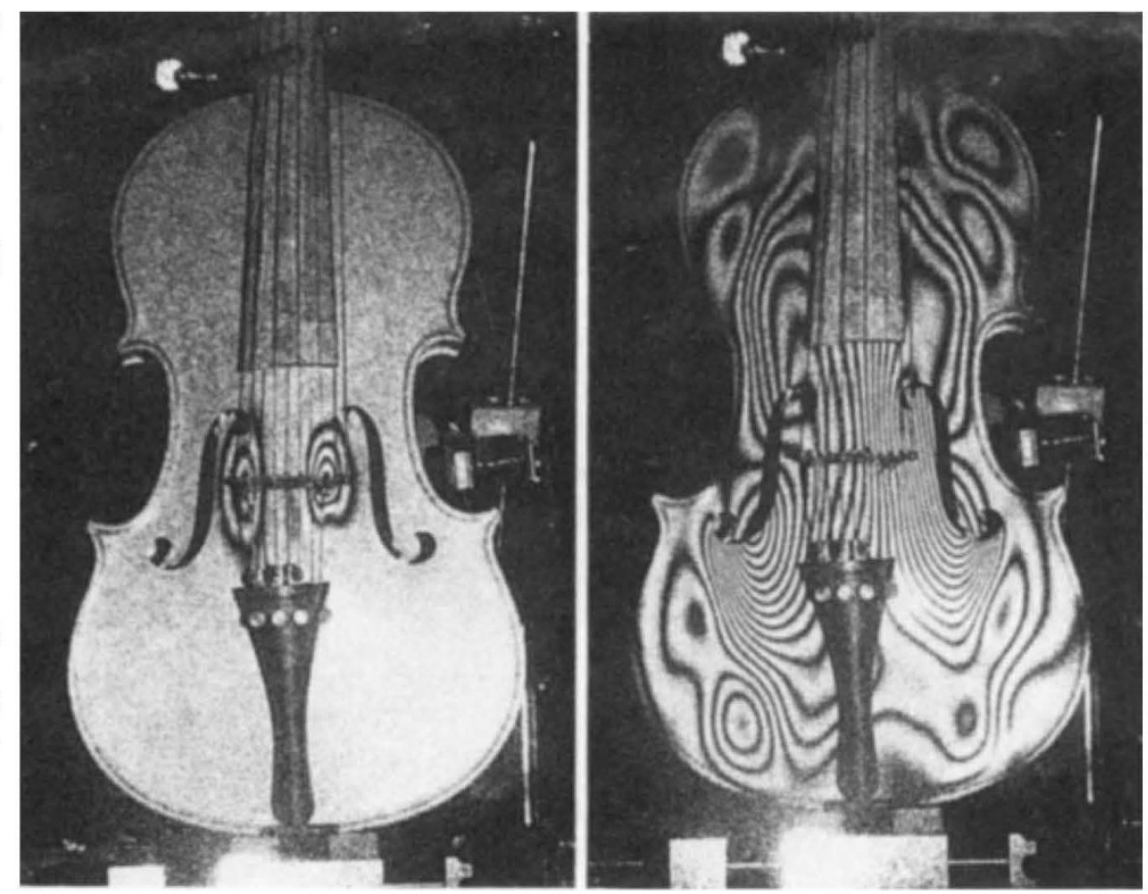

THE most characteristic part of a musical note, musicians hold, is the initial burst of sound: deprive a note of this initial waveform, and many instruments become indistinguishable. Nils-Erik Molin and colleagues (J. acoust. Soc. Am. 88, 2479-2481; 1990) have studied this moment for a violin by striking the instrument's 'bridge' (centre) with a light pendulum and, using laser interferometry, watching how the transient waveforms so stimulated propagate through its body. The figures above, taken 100 and 450 microseconds after impact, show the dipolar character of the waves generated at the two 'feet' of the bridge, and how the high frequencies quickly arrive at the 'f-holes' cut into the belly of the violin. The slight asymmetry in the dipolar waveform is attributable to subtle differences in the tension at each foot. 